RESEARCH ARTICLE

\title{
Lifestyle Intervention for Transplant Success (LIFTS) Mobile Health Wellness Program for Kidney Wait-Listed Patients: Development and Design
}

\author{
Sieverdes $\mathrm{JC}^{1^{*}}$, Bergamin $\mathrm{M}^{2}$, Chandler $\mathrm{J}^{1}$, Noltemeyer $\mathrm{Z}^{1}$, Moore $\mathrm{TL}^{3}$, Baliga $\mathrm{PK}^{4}$, Ruggi- \\ ero $\mathrm{KJ}^{1}$, Campbell $R \mathrm{C}^{4}$, Nemeth $L \mathrm{~S}^{1}$, Treiber $\mathrm{FA}^{1,2}$, Juergens $\mathrm{K}^{4}$ and Jenkins $\mathrm{CH}^{4}$ \\ ${ }^{1}$ College of Nursing, Medical University of South Carolina, SC, USA \\ ${ }^{2}$ Department of Medicine, Sport and Exercise Medicine Division, University of Padua, Padua, Italy \\ ${ }^{3}$ Donate Life South Carolina, Greenville, SC, USA \\ ${ }^{4}$ College of Medicine, Medical University of South Carolina, SC, USA
}

*Corresponding author: John C Sieverdes, PhD, College of Nursing, Medical University of South Carolina, 99 Jonathan Lucas St. MSC 160 Charleston, SC 29425-1600, USA, Tel: +843-792-1235, Fax:+843-792-2099, E-mail: sieverde@musc.edu

\begin{abstract}
Dialysis patients are at risk of attrition from Kidney Transplant (KT) wait lists due to sedentary lifestyle and poor nutritional adherence. This study tested the acceptability of several mobile Health (mHealth) intervention components for the development of a lifestyle program to support kidney wait-list patients.
\end{abstract}

Methods: A home-based, 3-month feasibility trial of a distance coaching $\mathrm{mHealth}$ program entitled Lifestyle Improvements for Transplant Success (LIFTS) was developed from interviews with 22 kidney wait-list dialysis patients. Program components of the LIFTS program include: 1) A walking program using Fitbit devices and app (Charge 2/Alta HR or latest model in line) to track steps on non-dialysis days, 2) Muscle strengthening using video education (links to YouTube $^{\mathrm{TM}}$ ), 3) Renal disease nutrition and physical education using weekly videos, 4) Nutrition tracking using MyFitnessPal app and 5) Weight/fluid variability tracking using Nokia (formerly Withings) Wi-Fi scales and inclusive smartphone app.

Activity steps and weight were remotely monitored in real-time and goals modified by an online coach every two weeks. Acceptability was primary assessed from exit interview content and quantified apps' measures to assess program components' use along with physical function and psychosocial changes.

Results: One White male (age 45) and 2 Black women (ages 24, 35) were recruited. Findings demonstrated: (1) Support of using Fitbits in walking program, but some wear ability/technical issues (i.e., $98 \%, 82 \%$, and $29 \%$ wear adherence), (2) Support using weight scales (mean $=2.54$ weigh-ins/week), (3) Nonadherence to suspension training protocols, suggesting need for a different approach, (4) Dietary videos were helpful, (5) The dietary tracking app was too burdensome and (6) Participants interested in more social elements and challenges. Overall, all participants felt the program improved their health.

Conclusions: Futher methodology refinement guided by dialysis patients to enhance acceptability is needed prior to a subsequent pilot study. Findings may be applicable in $\mathrm{mHealth}$ designs in highly fatigued patients.

\section{Keywords}

Dialysis, Fatigue, Transplantation, Mobile health, Physical activity, Nutrition, Telehealth

\section{Introduction}

Dialysis is a necessary treatment for over 678,000 patients suffering from End-Stage Renal Disease (ESRD) and over 90,000 on Kidney Transplant (KT) wait-lists in the US [1]. Although dialysis acts as a surrogate for kidney filtration, the treatment process negatively affects patients' health status and quality of life [2]. A common side effect of dialysis is persistent high fatigue levels (i.e., $60-97 \%$ of patient population) [3], which is higher than the general adult population [4]. Dialy-

Citation: Sieverdes JC, Bergamin M, Chandler J, Noltemeyer Z, Moore TL, et al. (2017) Lifestyle Intervention for Transplant Success (LIFTS) Mobile Health Wellness Program for Kidney Wait-Listed Patients: Development and Design. Int Arch Nurs Health Care 3:086. doi.org/10.23937/24695823/1510086

Received: September 02, 2017: Accepted: December 06, 2017: Published: December 08, 2017

Copyright: (C) 2017 Sieverdes JC, et al. This is an open-access article distributed under the terms of the Creative Commons Attribution License, which permits unrestricted use, distribution, and reproduction in any medium, provided the original author and source are credited. 
sis-associated fatigue is postulated as a primary driver of increased sedentary lifestyle behaviors that influence declining cardiovascular and muscular function [3-5]. Dialysis treatment filters amino acids and protein from the blood, which are necessary for maintaining extracellular fluid volume and muscle mass [6,7]. A lack of physiological muscular innervation combined with a less than adequate nutritionally-enriched humoral environment often results in a loss of muscle size and function. By breaking the cycle of this decline and sustaining or improving muscle function, medical providers can limit patient disability and death [8].

Many studies have found an increase in aerobic and anaerobic capacity in dialysis center settings through nurse and research staff mediated exercise programs either prior to or during dialysis [9-26]. Findings demonstrate increases in various biomechanist and physiological improvements in physical capacity [9-11]. Benefits of intradialytic exercise also result in increased urea clearance, reduced arterial stiffness and reduced risk of cardiac events [27-29]. Dialysis center-based physical activity (PA) intervention outcomes have shown mixed results, although most improve aerobic muscular function, increase muscular hypertrophy, increase strength, reduce cardiovascular disease risk factors [12-26] and increase quality of life (QOL) outcomes [11,24,30-32]. Although adoption and sustainability have been poor [33], the lack of participation may not have properly addressed patient specific issues such as fatigue, physical capabilities and the environment or the types of activities that may not be acceptable to dialysis patients $[4,34]$. There has been little research targeting lifestyle behaviors at home for kidney disease patients, but those incorporating both aerobic and resistance training have shown promise to increase PA and strength $[35,36]$. Less is known about the efficacy of these home-based program's impact on aerobic and anaerobic capacity but is an area of increasing interest [11,22].

Overall, the best treatment for patients with ESRD is a successful KT. Many patients with poor functional status or deterioration while waiting for a transplant can potentially benefit from interventions that focus on physical function. These would directly reduce CVD risk factors and aid ESRD patients in remaining eligible for a KT. mobile Health (mHealth) technology incorporating smartphone-based programs is a potential strategy for augmenting home-based program approaches for KT wait-list patients. mHealth technologies incorporating real-time sensor technologies have shown efficacious and sustainable results in self-managed behavior outcomes for ESRD patients, KT recipient groups, as well as other chronic disease conditions including diabetes, stroke and hypertension $[35,37,38]$. Using a mHealth approach to improve hemodialysis patients' health status has potential to be a low-cost and highly scalable delivery system to address patients' physical capacities while also reaching the $11.6 \%$ other patients undergoing home dialysis treatments who wouldn't be able to take part in center-based exercise programs [1,37]. However, the acceptability of smartphone-based programs and apps for self-management of PA and dietary conformance have not been empirically evaluated in KT wait-list patients and may present specific challenges.

Therefore, the purpose of this report is to detail the methodological development of a mHealth based wellness program for KT eligible ESRD patients. Using an iterative-staged, patient-centered, community driven, theory-based approach, the Lifestyle Intervention for Transplantation Success (LIFTS) program includes a formative evaluation, iterative preliminary testing, a proof of concept feasibility trial and a pilot trial. This report will describe development process and the feasibility trial of LIFTS to test the acceptability of the platform and provide feedback for the pilot study refinement.

\section{Methods and Materials}

\section{Overview}

The long-term goal of the LIFTS program is to be operationalized as a transplant center mHealth program designed to reach all KT eligible patients in a transplant center's service area. The overarching objective of LIFTS is to deliver a mHealth intervention that primarily supports the maintenance or increase in subjects' physical function (i.e., strength to ambulate, perform activities of daily living, etc.) and better control blood serum markers (i.e., through dietary and fluid conformance) necessary to sustain health thereby prolonging KT waitlist status. The development framework uses an iterative process to create and test the feasibility of the program prior to a pilot trial. This phase of the study uses the LIFTS program in a local 1-arm 30-person 3-month proof of concept feasibility trial. Increased PA and dietary self-management practices may serve to improve overall medical care treatment thus reducing cardiac, blood pressure, and metabolic related issues to support active KT wait-list status.

Primary outcomes include feasibility measures consisting of recruitment acceptability, program tolerability via retention rates, engagement in study device protocols, completing education sessions, compliance in entering weekly data in smartphone apps, acceptability measured through Likert scales on program components and the MARS mHealth acceptability scale (see detailed cut-points in measures section). Secondary outcomes include changes in physical functional testing, fatigue levels, improvements in PA, improvements in dietary control and the Malnutrition-Inflammation Score (MIS) index. Exploratory measures include conformance to standard patient blood serum/urine markers including albumin, creatinine, phosphorous and sodium from Nephrology/dialysis center reports. 


\section{Development}

Using a Community Based Participatory Research (CBPR) approach [39], stakeholders included doctoral-level behavioral scientists, transplant surgeons, nephrologists, Donate Life South Carolina leadership and nurse researchers. Stakeholder input directed the development of the LIFTS components and study protocol. Underlying behavioral theories included the Self-Determination Theory $[40,41]$ and Social Cognitive Theory to guide use of self-regulation and self-efficacy concepts during the program $[42,43]$. These theories formed the basis of the focus group questions during the formative phase [43] and were used to influence design of program components.

The initial development of the LIFTS program was based on the prior formative phase that investigated lifestyle habits, preferences and barriers KT wait-list patients experience if they were to engage in $\mathrm{mHealth}$ wellness programs [43]. In summary, the formative phase used 22 key informant interviews to identify ESRD patient physical capabilities, the utility of a smartphone platform for program delivery, current lifestyle patterns (i.e., current nutrition and exercise practices, fatigue, social support, perceived physical limitations, cultural/ environmental support, religious beliefs, motivations and accountability to remain healthy). Grounded theory based analyses [44] using transcripts and NVivo 10 (QSR International) resulted in preferences to engage in wellness programs outside the dialysis center setting on non-dialysis days due to concern about exercising in front of those who were unable or unwilling to participate [43]. Findings also showed positive reception to smartphone-based programs incorporating online video education, apps to log dietary data, communicating using texting (e.g., supportive motivational messages, program of the day, feedback, goal setting instructions), at home exercises, PA monitors and internet connected weight scales as long as training was provided to ease concerns [43].
Initial program development created a framework that interlinked existing sensor devices and apps (i.e., Fitbit, Nokia [formerly Withings], MyFitnessPal) monitored by a wellness coach. A panel of dialysis center nursing managers and two KT wait-listed dialysis patients met to review a framework of the program to elicit feedback. After refinement, small scale preliminary testing was used to test the LIFTS program concept over a 7-week period using three KT eligible volunteers to identify barriers, test communication flow, identify receptivity of equipment, and redefine training needs. Study activities were performed at Dialysis Center Incorporated (DCI) locations in Charleston County, South Carolina, USA. All study protocols were approval by MUSC and $D C l$ internal ethical review boards prior to initiation of research activities. Testing was conducted in 2014 with no reported adverse events. Afterward, iteration of LIFTS program components and intervention framework were finalized for the feasibility trail (Figure 1).

\section{LIFTS intervention program components}

The LIFTS system will utilize widely available consumer products and smartphone apps for the feasibility study. Primary components of the LIFTS program include: 1) A walking program using Fitbit devices and app (Charge 2/Alta HR or latest model in line) to track steps on non-dialysis days, 2) Muscle strengthening using video education (links to YouTube ${ }^{\mathrm{TM}}$ ), 3) Renal disease nutrition and physical education using weekly videos, 4) Nutrition tracking using MyFitnessPal app and 5) Weight variability tracking using Nokia Wi-Fi scales and Nokia's Health Mate smartphone app. Each component is discussed in detail in the following sections.

Walking program: A walking program will provide the basis for increasing PA. Following constructs of self-regulation and goal setting, subjects will be given Fitbit devices to track steps [45]. Studies have shown acceptable usage of Fitbit devices for recording steps and

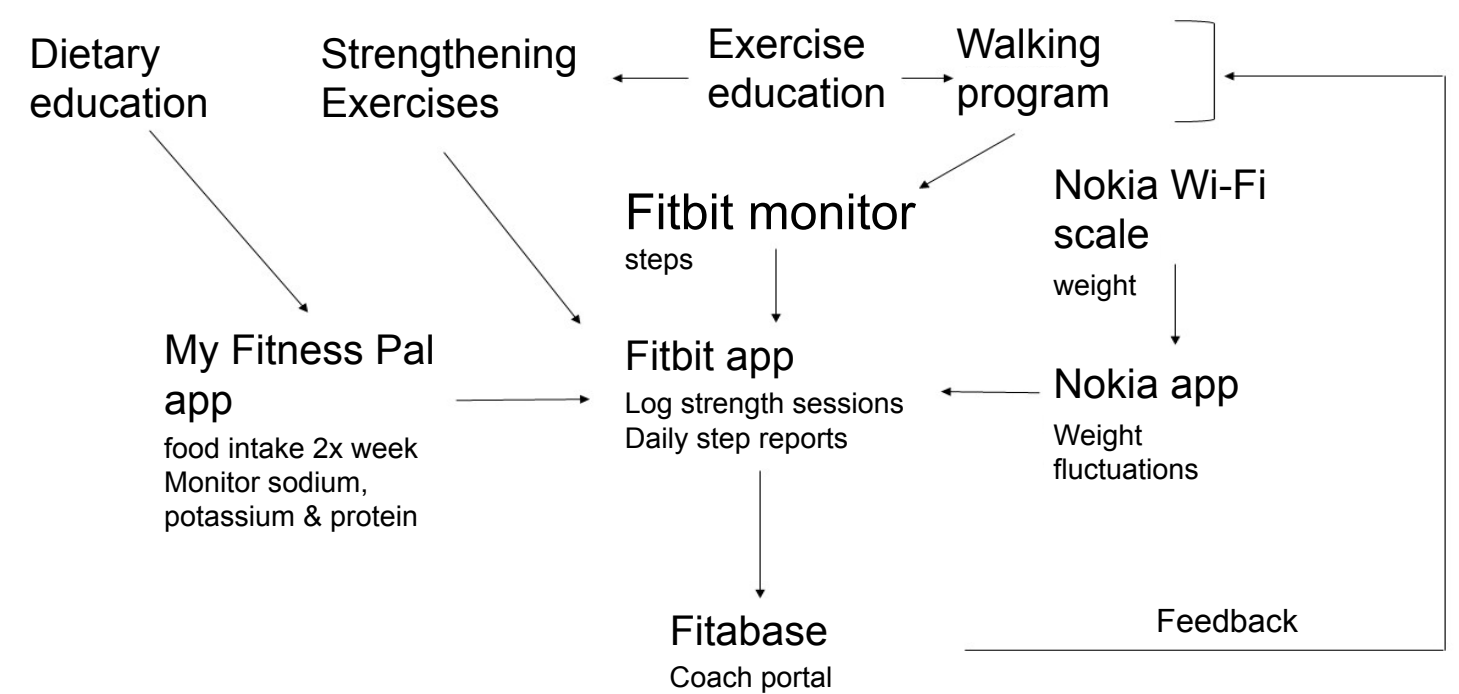

Figure 1: LIFTS information data capture and flow. 
their use in health promotion studies [46-51]. The walking program consists of scheduled exercise on three non-dialysis days and an overall weekly goal. Coaches set individual daily step goals in collaboration with subjects and are modified every two weeks ten-minutes per non-dialysis day would be the initial target duration for each walk that progresses through the program. Alterations to the initial duration can be individualized during the orientation session. Reminders for walks are delivered via SMS text messages to subjects on walking days. If subjects meet their step goal $75 \%$ of the time or greater, then step goals would increase by $10 \%$. If subjects meet their step goal less than $25 \%$ of the time, then step goals decrease by $10 \%$ with behavioral trouble shooting at the time of contact.

Muscle strengthening: Muscular strengthening activities will use body weight exercises using body weight and common household items. Exercises will be modified to the specific needs and limitations of each participant (i.e., one-arm exercises, etc.) by the wellness coach, with specific attention regarding dialysis port sites, where only range of motion may be emphasized. Subject preference will dictate whether strength sessions are used in conjunction with the walking program or performed at another time. Strengthening programs' exercises, sets, and target reps will be sent using SMS text reminders containing hyperlinks to example videos on a private YouTube ${ }^{\mathrm{TM}}$ video streaming channel each morning on three predetermined non-dialysis days. Two to five exercises using one to three sets will be prescribed using traditional progressive overload principles, increasing the number of exercises and/or sets in successive weeks [52]. Examples of exercises could include chair stands, body squats, wall push-ups, 1-arm wall/table push-ups, milk gallon curls, chair-supported leg raises and 1-arm rows using weighted grocery bags, etc. If the participant has difficulty performing exercises, modification of exercise selection or range of motion would be determined through conversations with the coach every two weeks. At two months, subjects would suggest their own exercise plans, thereby increasing self-efficacy to develop their own routines. Subjects would be asked to record strengthening exercise sessions in the Fitbit smartphone app as a completed session.

Nutrition and exercise education: Renal nutrition and exercise education scripts have been developed in collaboration with a Registered Dietitian specializing in the care of renal disease patients and an Exercise Physiologist. Scripts were used to develop a series of short, one-minute informational videos that are delivered once a week using SMS text messaging and hyperlinks. Videos are hosted on YouTube ${ }^{\mathrm{TM}}$. Fifteen videos have been developed and include grocery store footage with voice over for the following titles: 1) Controlling your fluid, 2) Grocery lists - planning ahead, 3) Supermarket Shopping tips, 4) Low sodium seasonings, 5) Balancing your protein, 6) Low potassium fruits and vegetables, 7) Food and drink portion control, 8) Reading food labels: Potassium and phosphorous, 9) All about sodas, 10) Choosing the right walking shoes, 11) How intense should you exercise, 12) Realistic expectations, 13) Rewarding yourself, 14) Find some fun in your exercise, and 15) Get a walking buddy.

Nutrition and weight tracking: The MyFitnessPal app will be downloaded to each subjects' smartphone for tracking food intake and data linked to the Fitbit app. Subjects will be trained and asked to enter their food intake two days a week including one weekday and one weekend day. Subjects will monitor their nutrient intake including sodium, potassium, and protein through app reports and asked to make adjustments according to their physician's recommended tolerance range.

Wi-Fi connected Nokia weight scales would be supplied for weight monitoring [53]. Subjects will be asked to weigh themselves every morning and night to assess their weight fluctuations. Weight fluctuation viewed in the Nokia My Health Mate app is intended to give rough estimates of fluid variability and provide self-regulation of nutritional and fluid intake.

Coach interactions: A Certified Personal Trainer will serve as the wellness coach and communicate by text and phone calls to each participant throughout the study period. The coach will monitor program adherence and Fitbit use status during each week of the program. Program fidelity will be monitored in real-time using Fit a base (Small Steps Labs, LLC.) that aggregates data from all study apps into a downloadable reporting form and Fitbit status dash board. Fitabase software imports Fitbit steps, calorie intake via MyFitnessPal, exercise sessions and Nokia scale measurement data (i.e., weight, body fat \%) from the devices and provides feedback for device usage to the wellness coach (i.e., Fitbit battery charging state, last time synced, etc.). For subject non adherence greater than 3 days, a text message will be sent followed by a phone call on the fifth day to troubleshoot issues. Subjects will be instructed to notify the wellness coach if devices malfunction, apps disconnect, or if devices are lost or broken.

Motivational SMS text messages will be sent by the coach once per week to provide feedback on program adherence to the study protocol. Coaching sessions using phone calls will be given once a week for the first 2 weeks then every other week thereafter. Coaching sessions will be used to assess barriers, provide feedback for exercise selection and change goals. Each subject's account will also be connected through the social feature within Fitbit's companion app to encourage social support between subjects. They will be allowed to invite other friends as they desire.

\section{Sample and recruitment}

Thirty subjects will be recruited for the one arm fea- 
sibility study. The sample size is not powered but reflect sizes chosen (i.e., ranges between 23-50) based upon recommendations by Sims \& Lewis for feasibility studies not to address power but to address precision and efficiency [54,55]. Dialysis Center Incorporated (DCI) and the Medical University of South Carolina (MUSC) Transplant Center are the study partners and serve as the environment for the study. Potential study subjects will be identified from MUSC Transplant Center KT wait-list records and medically approved by physicians from the Department of Transplant Surgery. Inclusion criteria consists of wait-listed dialysis patients between ages 18 and 65 , male or female, of any racial groups, who take hemodialysis at $\mathrm{DCl}$ centers or take home dialysis treatments in Charleston county, South Carolina, USA. Although we foresee most subjects will have their own smartphones, smartphones will be made available to those without their own device. Exclusion criteria consists of inactive status on the KT wait-list, persons unable to operate a smartphone effectively after training and mental health issues identified by the study physicians.

\section{Protocol}

Potential subjects from $\mathrm{DCl}$ dialysis centers will be approached by $\mathrm{DCl}$ nursing staff, and if interested, followed up on-site with research staff to review study protocol and complete informed consent procedures. Orientation for subjects will take place in a private conference room at each $\mathrm{DCl}$ center prior to dialysis treatment or on a non-dialysis day. Home dialysis potential subjects will be called by a transplant center coordinator and if interested, use the next on-site MUSC evaluation appointment for the orientation session in a private conference room. Orientation sessions will last approximately one hour and include baseline psychosocial and physiological measures, reported activity history, step and weight goal setting, training for each of the apps used in the LIFTS components, hands-on dietary and exercise entry, dietary portion size training, and hands-on body weight exercise selection and training. Post-study (i.e., at 3 months) in-person follow-up will be performed to measure psychosocial and physiological measures. $\mathrm{DCl}$ centers and patient $\mathrm{Ne}-$ phrology offices will be contacted to obtain blood serum/ urine measures for pre-post analyses.

\section{Measures}

Demographic measures: Baseline demographics measures include age, gender, race, height (i.e., self-reported), weight (i.e., dry weight from center/clinic scale), months on dialysis, prior and current activity status and social living condition (i.e., living alone, or with others?). Waist and hip circumference are measured using standard protocols with three readings for each measure with the two closest values averaged.

\section{Primary outcomes:}

Acceptability measures: Primary outcomes include feasibility measures consisting of recruitment acceptabil- ity (\% of participants approached participating [acceptability target $\geq 85 \%$ ]), tolerability (drop out \% [acceptability target $\leq 10 \%]$ ), engagement (\% increasing and sustaining activity levels $\geq 15 \%$ via step counts [acceptability target $\geq 75 \%$ ], \% of video modules completed [acceptability target $80 \%$ videos watched per person with $\geq 75 \%$ average compliance, \% completion of dietary assessment [acceptability target $\geq 75 \%$ ]) and the Mobile Application Rating Scale (MARS). MARS includes 4 objective scales that measure engagement, aesthetics, functionality and information quality along with a subjective quality scale [56]. An average rating of $80 \%$ on all MARS scales is targeted. Additionally, quantitative measures relating to device/components will use 0-10 Likert scales (i.e., $0=$ "do not agree at all"...10 = "strongly agree") to evaluate the following questions: "Did you find the device/component important to meet your goals?", "Was the device/component easy to integrate in your daily life and easy to use?" and "Moving forward, would you continue to want to use this device/component?". A brief end of study interview using open-ended questions will cover other aspects of the program including program set-up, technology barriers, log-ins to apps, most and least used components of the intervention, perceptions on the amount and value of feedback received [57]. Exit interview questions incorporated established concepts regarding utilization and participation will be pretested using an advisory panel consisting of qualitative analysts, psychologists and mHealth researchers $[57,58]$.

\section{Secondary outcomes:}

Physiological measures: Subjects will complete battery of physiological tests pre- and post-study including the Timed Up and Go Test (TUG), 30-second Chair Stand Test and a hand-grip strength test (i.e. Grip Strength South Hampton protocol [59] using a Jamar Dynamometer) to assess physical function [60].

Psychosocial measures: Psychosocial measures include Self-Efficacy (SE) scales for PA and a modified derivation for $S E$ of strength training using the Exercise SE Scale (ESES) [61]. SE for PA and strength training is based on a 10-item 5-point Likert scale. Nutrition adherence will use the Renal Adherence Behavior Questionnaire (RABQ) scale and it's subscales on self-care [62] with measures based on 5 -factor analysis of 25 items pertaining to renal nutritional adherence. Fatigue will be measured using the Functional Assessment of Chronic Illness Therapy Fatigue Scale (FACIT-F). FACIT-F is a 13-question, 0-52 ranged scale where higher responses represent lower fatigue with general population median score $=47$ [63]. Lastly, the MIS scale, one of the secondary outcomes, will be used to measure malnutrition and inflammatory conditions which is commonly used as a risk metric for protein energy muscle wasting [64].

\section{Exploratory outcomes:}

Serum measures: Patient data will be sourced from patients' dialysis centers and nephrology office records. 
Specific measures include serum or urine albumin, creatinine, phosphorous, and sodium. Percent change in weeks of conformance, absolute change, and conformance to $\geq$ $75 \%$ for each subject per individualized physician dictated goal will be analyzed. Records will include the three months prior to the study and all study months.

Fidelity measures: PA metrics obtained by the Fitbit devices included wear days, steps, and Fitbit activity minutes (sedentary, light, fairly active and very active minutes). Average steps and minutes will be calculated using a minimum of four days of wear to be considered a valid week. Although Fitbit devices do not report wear time, subjects are instructed to wear the device 24-hours a day except during activities involving submerging the device in water, showering, or bathing unless current models are waterproof. Usage will be assessed through days of wear divided by the expected number of days. Nokia weight scale metrics include the number of weigh-ins and recorded weights. Acceptable fidelity is set at $\geq 75 \%$. Multiple weigh-ins on the same day will be averaged with differences in morning to night plotted. Dietary logging through the MyFitnessPal app will be assessed by counting data entry days divided by the number of expected days.

\section{Analysis}

Baseline demographic variables will be reported using mean and Standard Deviations (SD) for continuous and percentages for categorical variables. Outcomes variables will be tested for normality using Shapiro-Wilks testing. Student's paired T-testing using a significance of $p=0.05$ will be used to test significance differences pre-to-post intervention for continuous variables and Chi-square tests for categorical variables. Nonparametric tests will be substituted if normality is not met. Adherence measures will be compared to their corresponding targets. Fitbit variables include average weekly steps and weekly differences between dialysis days and non-dialysis (i.e., non-dialysis days average dialysis days average). Nokia scale weigh-ins and morning to night differences will be extracted from Fitabase data. Fidelity measures from Fitbit, My Health Mate and MyFitnessPal apps' adherence data will be extracted through Fit a base and assessed using \% adherence to the protocol. Responses from the exit interviews will be recorded and extracted in the qualitative analysis along with average quantitative scores for each device/ component question. Each program component will be ranked by using positive or negative responses with aggregated needs and wants lists to be deliberated during future refinement for the pilot study.

\section{Results}

Our preliminary developmental results using the test subjects informed increased training needs regarding the MyFitnessPal and Fitbit apps. It is suggested that subjects need hands-on training and show research staff they can perform the activities to prove they can complete intervention protocols. During the testing, suspension training straps were originally utilized with modified exercise videos with limited instruction during orientation. Training straps were underutilized during follow-up noting concern with dialysis port sites, unfamiliarity with the exercise modality, low motivation and increased training needs. Therefore, the stakeholders decided to remove the suspension training straps and to simplify and reduce the number of strengthening exercises. Other notable lessons learned established that orientation should be performed prior to dialysis treatment or on a non-dialysis day since patients were more fatigued after dialysis. It was also found that PA monitors that are wrist worn incorporating a watchstyle clasp would provide the best method to secure the device and reduce the potential of lost equipment. Lastly, patients may need to have a dialysis center contact to assist in technology troubleshooting to login and access apps if conditions requiring new equipment or if smartphones are replaced. These findings were used to reiterate the approach, methods, communication, and components of the presented methodology.

Results of the feasibility study will seek to ascertain if the program is acceptable and tolerable to ESRD patients in its current iteration. Feedback and lessons learned during the trial will inform training needs and refinement of the LIFTS program. Secondary outcomes will be used to calculate a properly powered sample size for use in the subsequent pilot study.

\section{Discussion}

This study defines the methodology of a mHealth wellness program for ESRD KT waitlist patients. In addition, the development process uncovered issues researchers may encounter when executing mHealth studies highlighting the need to propose test case scenarios during program development. Results of the proposed feasibility study will inform clinical researchers about the acceptability of a distance-based, low contact wellness program for ESRD patients that may be generalizable to other patients with high levels of fatigue.

We found several technology-related issues for this mHealth study that require careful planning to ensure device compatibility, training needs and study robustness. For instance, firmware updates could disconnect devices from users' smartphones requiring re-installation of software or re-pairing a device. Plans in the protocol should include careful planning to support subject troubleshooting for such a case scenario. We also initially had issues with compatibility concerns to connect study devices to subjects' smartphones. At the time of initial development (i.e., 2014) smartphone adoption was reported at $53 \%$ when only six compatible devices existed to connect to Fitbit/Internet connected scales [65]. Currently, over 200 smartphone devices are now compatible with the proposed study devices and smartphone adoption has increased to $77 \%[45,65]$. This al- 
leviated technology availability concerns that were responsible for potential lower adoption rates. There was also issue of subjects not knowing how to connect devices to their smartphones due to low technical experience using apps. Establishing training aids and handson examples letting the subject connect and disconnect devices is warranted to drive device ownership and ability to troubleshoot issues during this study and other mHealth studies.

Several limitations of this study should be addressed. The length of the proposed study may be too short to elicit a physical response thus reducing the impact of physiological findings. It may also be implicit that the LIFTS intervention may only conserve strength and physical function rather than improving outcomes. The design of the study does not include a control group limiting the power of our findings, though is justified due to being predominantly focused on acceptability outcomes. The intervention design is also multi-component so therefore we will be unable to differentiate which program component may provide the largest impact on the measured outcomes. Although these limitations are inherent in the study design, there are several strengths including a patient and provider centered design, a mixed methods approach to evaluate program acceptability and patient-centered and clinically relevant outcomes driven through community-based research framework.

\section{Conclusion}

This study will determine the initial feasibility of a smartphone-based approach to improve PA and nutrition conformance in ESRD patients on the KT waitlist. Components such as a walking program utilizing PA monitors, tracking body weight fluctuations using internet connected scales, and delivering education content using short video segments may be a method to introduce a novel approach to reach ESRD patients throughout a transplant center service area without the need for frequent in-person contact. Results will inform usage, perceptions, needs, training and additional refinement to deliver an efficacious program to improve patient care. Although LIFTS is tailored to ESRD patients on the KT wait-list, derivations may include non-waitlisted ESRD patients, KT patients and CKD patients. If an mHealth approach can increase or maintain physical function and provide better health for ESRD patients as with dialysis center programs [26], then this may provide a widely disseminable and low cost option to enhance patient health.

\section{References}

1. USRDS (2016) Annual Report.

2. Tonelli M, Wiebe N, Knoll G, Bello A, Browne S, et al. (2011) Systematic review: Kidney transplantation compared with dialysis in clinically relevant outcomes. Am J Transplant 11: 2093-2109.
3. Horigan AE (2012) Fatigue in hemodialysis patients: A review of current knowledge. J Pain Symptom Manage 44: 715-724.

4. Jhamb M, Weisbord SD, Steel JL, Unruh M (2008) Fatigue in patients receiving maintenance dialysis: A review of definitions, measures, and contributing factors. Am J Kidney Dis $52:$ 353-365.

5. Chao CT, Huang JW, Chiang CK, COGENT (COhort of GEriatric Nephrology in NTUH) study group (2016) Functional assessment of chronic illness therapy-the fatigue scale exhibits stronger associations with clinical parameters in chronic dialysis patients compared to other fatigue-assessing instruments. PeerJ 4: e1818.

6. Carrero JJ, Stenvinkel P, Cuppari L, Ikizler TA, Kalantar-Zadeh K, et al. (2013) Etiology of the protein-energy wasting syndrome in chronic kidney disease: A consensus statement from the International Society of Renal Nutrition and Metabolism (ISRNM). J Ren Nutr 23: 77-90.

7. Lenk K, Schuler G, Adams V (2010) Skeletal muscle wasting in cachexia and sarcopenia: Molecular pathophysiology and impact of exercise training. J Cachexia Sarcopenia Muscle 1: 9-21.

8. Johansen KL, Kaysen GA, Dalrymple LS, BA Grimes, DV Glidden, et al. (2013) Association of physical activity with survival among ambulatory patients on dialysis: The Comprehensive Dialysis Study. Clin J Am Soc Nephrol 8: 248-253.

9. Johansen $\mathrm{KL}$ (2008) Exercise and dialysis. Hemodial Int 12: $290-300$.

10. Jung TD, Park SH (2011) Intradialytic exercise programs for hemodialysis patients. Chonnam Med J 47: 61-65.

11. Painter P, Carlson L, Carey S, Paul SM, Myll J (2000) Physical functioning and health-related quality-of-life changes with exercise training in hemodialysis patients. Am J Kidney Dis 35: 482-492.

12. Bohm CJ, Ho J, Duhamel TA (2010) Regular physical activity and exercise therapy in end-stage renal disease: how should we move forward? J Nephrol 23: 235-243.

13. Bohm J, Monteiro MB, Thome FS (2012) Effects of aerobic exercise during haemodialysis in patients with chronic renal disease: a literature review. J Bras Nefrol 34: 189-194.

14. Boyce ML, Robergs RA, Avasthi PS, Roldan C, Foster A, et al. (1997) Exercise training by individuals with predialysis renal failure: Cardiorespiratory endurance, hypertension, and renal function. Am J Kidney Dis 30: 180-192.

15. Castaneda C, Gordon PL, Uhlin KL, Levey AS, Kehayias JJ, et al. (2001) Resistance training to counteract the catabolism of a low-protein diet in patients with chronic renal insufficiency. A randomized, controlled trial. Ann Intern Med 135: 965-976.

16. Cheema B, Abas H, Smith B, O'Sullivan A, Chan M, et al. (2007) Randomized controlled trial of intradialytic resistance training to target muscle wasting in ESRD: The Progressive Exercise for Anabolism in Kidney Disease (PEAK) study. Am J Kidney Dis 50: 574-584.

17. Cheema B, Abas H, Smith B, O'Sullivan A, Chan M, et al. (2007) Progressive exercise for anabolism in kidney disease (PEAK): A randomized, controlled trial of resistance training during hemodialysis. J Am Soc Nephrol 18: 15941601.

18. Chen JL, Godfrey S, Ng TT, Moorthi R, Liangos O, et al. (2010) Effect of intra-dialytic, low-intensity strength training on functional capacity in adult haemodialysis patients: A randomized pilot trial. Nephrol Dial Transplant 25: 1936-1943. 
19. Clyne N (2004) Physical working capacity and muscle strength in chronic renal failure are improved by exercise. Lakartidningen 101: 4111-4115.

20. DePaul V, Moreland J, Eager T, Clase CM (2002) The effectiveness of aerobic and muscle strength training in patients receiving hemodialysis and EPO: A randomized controlled trial. Am J Kidney Dis 40: 1219-1229.

21. Johansen $\mathrm{KL}$ (2007) Exercise in the end-stage renal disease population. J Am Soc Nephrol 18: 1845-1854.

22. Koh KP, Fassett RG, Sharman JE, Coombes JS, Williams AD (2010) Effect of intradialytic versus home-based aerobic exercise training on physical function and vascular parameters in hemodialysis patients: A randomized pilot study. Am J Kidney Dis 55: 88-99.

23. Konstantinidou E, Koukouvou G, Kouidi E, Deligiannis A, Tourkantonis A (2002) Exercise training in patients with end-stage renal disease on hemodialysis: Comparison of three rehabilitation programs. J Rehabil Med 34: 40-45.

24. Lo CY, Li L, Lo WK, Chan ML, So E, et al. (1998) Benefits of exercise training in patients on continuous ambulatory peritoneal dialysis. Am J Kidney Dis 32: 1011-1018.

25. Segura-Orti E, Kouidi E, Lison JF (2009) Effect of resistance exercise during hemodialysis on physical function and quality of life: Randomized controlled trial. Clin Nephrol 71: $527-537$

26. Orcy RB, Dias PS, Seus TL, Barcellos FC, Bohlke M (2012) Combined resistance and aerobic exercise is better than resistance training alone to improve functional performance of haemodialysis patients-results of a randomized controlled trial. Physiother Res Int 17: 235-243.

27. Cooke AB, Ta V, lqbal S, Gomez YH, Mavrakanas T, et al. (2017) The Impact of intradialytic pedaling exercise on arterial stiffness: A pilot randomized controlled trial in a hemodialysis population. Am J Hypertens.

28. Brown PDS, Rowed K, Shearer J, MacRae JM, Parker K (2017) Impact of intradialytic exercise intensity on urea clearance in hemodialysis patients. Appl Physiol Nutr Metab 29: $1-4$.

29. Isnard-Rouchon M, Coutard C (2017) Exercise as a protective cardiovascular and metabolic factor in end stage renal disease patients. Nephrol Ther 13: 544-549.

30. Giannaki CD, Hadjigeorgiou GM, Karatzaferi C, Maridak MD, Koutedakis $Y$, et al. (2013) A single-blind randomized controlled trial to evaluate the effect of 6 months of progressive aerobic exercise training in patients with uraemic restless legs syndrome. Nephrol Dial Transplant 28: 28342840.

31. de Lima MC, Cicotoste Cde L, Cardoso Kda S, Forgiarini LA Jr, Monteiro MB, et al. (2013) Effect of exercise performed during hemodialysis: Strength versus aerobic. Ren Fail 35: 697-704.

32. Tentori F, Elder SJ, Thumma J, Pisoni RL, Bommer J, et al. (2010) Physical exercise among participants in the Dialysis Outcomes and Practice Patterns Study (DOPPS): Correlates and associated outcomes. Nephrol Dial Transplant 25: 3050-3062.

33. Manfredini F, Mallamaci F, Catizone L, Zoccali C (2012) The burden of physical inactivity in chronic kidney disease: Is there an exit strategy? Nephrol Dial Transplant 27: 2143-2145.

34. Delgado C, Johansen KL (2012) Barriers to exercise participation among dialysis patients. Nephrol Dial Transplant 27: 1152-1157.
35. Hiraki K, Shibagaki Y, Izawa KP, Hotta C, Wakamiya A, et al. (2017) Effects of home-based exercise on pre-dialysis chronic kidney disease patients: A randomized pilot and feasibility trial. BMC Nephrol 18: 198.

36. Heiwe S, Jacobson SH (2014) Exercise training in adults with CKD: A systematic review and meta-analysis. Am J Kidney Dis 64: 383-393.

37. Lai J, Porter J (2015) Dietary mobile apps and their effect on nutritional indicators in chronic renal disease: A systematic review. Nephrology (Carlton).

38. Hamine S, Gerth-Guyette E, Faulx D, Green BB, Ginsburg AS (2015) Impact of mHealth chronic disease management on treatment adherence and patient outcomes: A systematic review. J Med Internet Res 17: e52.

39. (2011) Principles of Community Engagement. ( $\left.2^{\text {nd }} e d n\right)$, $\mathrm{NIH}$, Washington, DC.

40. Deci EL, Ryan RM (1985) Intrinsic motivation and self-determiation in human behavior. Plenum Press, New York.

41. Ryan RM, Deci EL (2000) Self-determination theory and the facilitation of intrinsic motivation, social development, and well-being. Am Psychol 55: 68-78.

42. Bandura A (1986) Social foundations of thought and action: A social cognitive theory. Prentiss-Hall, Englewood Cliffs, NJ.

43. Sieverdes JC, Raynor PA, Armstrong T, Jenkins $\mathrm{CH}$, Sox LR, et al. (2015) Attitudes and perceptions of patients on the kidney transplant waiting list toward mobile health-delivered physical activity programs. Prog Transplant 25: 26-34.

44. Strauss A, Corbin J (1998) Basics of qualitative research: Grounded theory procedures and techniques. Sage Publications, San Francisco.

45. (2017) Fitbit device description web-page.

46. Chu AH, Ng SH, Paknezhad M, Gauterin A, Koh D, et al. (2017) Comparison of wrist-worn Fitbit Flex and waist-worn ActiGraph for measuring steps in free-living adults. PLoS One 12: e0172535.

47. Imboden MT, Nelson MB, Kaminsky LA, Montoye AH (2017) Comparison of four Fitbit and Jawbone activity monitors with a research-grade ActiGraph accelerometer for estimating physical activity and energy expenditure. $\mathrm{Br} \mathrm{J}$ Sports Med.

48. Middelweerd A, HP VDP, A VANH, Twisk JWR, Brug J, et al. (2017) A Validation Study of the Fitbit one in daily life using different time intervals. Med Sci Sports Exerc 49: 1270-1279.

49. Cadmus-Bertram L, Marcus BH, Patterson RE, Parker BA, Morey BL (2015) Use of the Fitbit to measure adherence to a physical activity intervention among overweight or obese, postmenopausal women: Self-monitoring trajectory during 16 weeks. JMIR Mhealth Uhealth 3: e96.

50. Diaz KM, Krupka DJ, Chang MJ, Peacock J, Ma Y, et al. (2015) Fitbit@: An accurate and reliable device for wireless physical activity tracking. Int J Cardiol 185: 138-140.

51. Wang JB, Cadmus-Bertram LA, Natarajan L, White MM, Madanat H, et al. (2015) Wearable Sensor/Device (Fitbit One) and SMS text-messaging prompts to increase physical activity in overweight and obese adults: A randomized controlled trial. Telemed J E Health 21: 782-792.

52. American College of Sports Medicine (2009) American College of Sports Medicine position stand. Progression models in resistance training for healthy adults. Med Sci Sports Exerc 41: 687-708. 


\section{3. https://health.nokia.com/in/en/}

54. Sim J, Lewis M (2012) The size of a pilot study for a clinical trial should be calculated in relation to considerations of precision and efficiency. J Clin Epidemiol 65: 301-308.

55. Julious SA (2005) Sample size of 12 per group rule of thumb for a pilot study. Pharm Stat 4: 287-291.

56. Stoyanov SR, Hides L, Kavanagh DJ, Zelenko O, Tjondronegoro $D$, et al. (2015) Mobile app rating scale: A new tool for assessing the quality of health mobile apps. JMIR Mhealth Uhealth 3: e27.

57. Bowen DJ, Kreuter M, Spring B, Cofta-Woerpel L, Linnan L, et al. (2009) How we design feasibility studies. Am J Prev Med 36: 452-457.

58. Riley-Jacome M, Gallant MP, Fisher BD, Gotcsik FS, Strogatz DS (2010) Enhancing community capacity to support physical activity: The Development of a community-based indoor-outdoor walking program. J Prim Prev 31: 85-95.

59. Roberts HC, Denison HJ, Martin HJ, Patel HP, Syddall H, et al. (2011) A review of the measurement of grip strength in clinical and epidemiological studies: Towards a standardised approach. Age Ageing 40: 423-429.
60. Segura-Orti E, Martinez-OImos FJ (2011) Test-retest reliability and minimal detectable change scores for sit-tostand-to-sit tests, the six-minute walk test, the one-leg heel-rise test, and handgrip strength in people undergoing hemodialysis. Phys Ther 91: 1244-1252.

61. Kroll T, Kehn M, Ho PS, Groah S (2007) The SCI Exercise Self-Efficacy Scale (ESES): Development and psychometric properties. Int J Behav Nutr Phys Act 4: 34.

62. Rushe H, McGee HM (1998) Assessing adherence to dietary recommendations for hemodialysis patients: The Renal Adherence Attitudes Questionnaire (RAAQ) and the Renal Adherence Behaviour Questionnaire (RABQ). J Psychosom Res 45: 149-157.

63. Webster K, Cella D, Yost K (2003) The Functional Assessment of Chronic Illness Therapy (FACIT) measurement system: Properties, applications, and interpretation. Health Qual Life Outcomes 1: 79.

64. Perez Vogt B, Costa Teixeira Caramori J (2016) Are nutritional composed scoring systems and protein-energy wasting score associated with mortality in maintenance hemodialysis patients? J Ren Nutr 26: 183-189.

65. (2017) Mobile Fact Sheet. Pew Research Center: Internet \& Technology. 